\title{
Formen der Dezentralisierung: Auswirkungen tarifpolitischer Entwicklungen auf die Effektivität der Betriebsratsarbeit
}

Martin Behrens

Dezentralisierung ist eines der wesentlichen Kennzeichen des Wandels des deutschen Tarifsystems. Während bislang die Auswirkungen auf die gewerkschaftliche Tarifpolitik im Zentrum des Interesses standen, wendet sich die folgende Analyse den Konsequenzen für die betriebliche Mitbestimmung zu. Mit Rückgriff auf die Daten der WSI-Befragung von Betriebsräten 2004/05 werden die Folgen unterschiedlicher Dezentralisierungsformen für den Erfolg der Betriebsratsarbeit analysiert. ${ }^{1}$

\section{Wandel der Dezentralisierungslogik}

Spätestens seit den frühen 1980er Jahren verdichteten sich Hinweise darauf, dass es in einer Vielzahl entwickelter kapitalistischer Ökonomien zur Dezentralisierung von Tarifbeziehungen gekommen ist. Hierbei geht es nicht allein um eine Verlagerung formaler Tarifstrukturen in Richtung dezentraler Einheiten, beobachtet wurden auch Veränderungen im Bereich der informellen Tarifpraktiken (Katz 1993).

Nun ist Dezentralisierung der Tarifbeziehungen aus gewerkschaftlicher Sicht weder ein neuer noch ein zwangsläufig dramatisierungswürdiger Tatbestand. So konnte Wittemann (1994) in seiner Analyse der Tarifpolitik der IG Metall der 1960er Jahre zeigen, dass bei Ford eine bewusste Strategie der Verbetrieblichung der Tarifbeziehungen als Ansatzpunkt für eine Strategie zum Ausbau der gewerkschaftlichen Handlungsmacht gesehen wurde. Wie dieses Beispiel bereits andeutet, verbieten sich bezüglich der Zentralität von Tarifpolitik vorschnelle Zuordnungen, wonach Gewerkschaften per se als Verfechter einer zentralisierten Tarifpolitik zu gelten hätten. In umgekehrter Perspektive gilt Ähnliches für die Arbeitgeberseite. Wie Swensons historische Analyse der schwedischen Tarifpolitik und -strukturen belegt, schienen die schwedischen Arbeitgeber keineswegs auf eine reine Dezentralisierungspolitik festgelegt zu sein. Schließlich waren es an erster Stelle die besonderen Arbeitsmarktinteressen des Arbeitgeberlagers, welche ursprünglich zur Zentralisierung der dortigen Tarifbeziehungen führten (Swenson 2002).
Seit Beginn der 1980er Jahre haben sich allerdings in vielen entwickelten Ökonomien die Interessenlagen bezüglich der Dezentralisierung neu ausgerichtet. Mit weiterer Öffnung der Märkte und einer teils aggressiv vorgetragenen Deregulierungspolitik manch konservativer Regierung scheint Dezentralisierung ihre Unschuld eingebüßt zu haben. So argumentieren Ferner/Hyman (1992, S. XX), dass neue Formen der Dezentralisierung eher Resultat von besonderen Strategien und Erfordernissen der Arbeitgeberseite sind und wohl kaum mehr als Ausdruck gewerkschaftlicher Verhandlungsmacht $\mathrm{zu}$ interpretieren seien. Befunde aus der vergleichenden Industrial Relations Forschung scheinen die These von Ferner/Hyman weitgehend zu bestätigen: Massive Dezentralisierung von Tarifstrukturen ergaben sich in Schweden (Swenson/Pontusson 2000), Großbritannien (Howell 1999, S. 37f.) sowie in den USA (Kochan et al. 1986, S. 65ff.). Allerdings ist die Dezentralisierung der Tarifbeziehungen nicht nur eine Frage der Radikalität und der Geschwindigkeit, sondern stets auch abhängig vom Ausgangsniveau, von dem aus Dezentralisierung wirksam wird. Wie die Fälle Schwedens und der USA belegen, kommt Dezentralisierung zu grundlegend unterschiedlichen Ergebnissen, je nach dem, ob diese bei nationalen, übersektoriellen Tarifverhandlungen (Schweden) oder bei betrieblichen Tarifsystemen (USA) ansetzt. Während in Schweden Branchentarifverträge auch nach der Ablösung nationaler Tarifverhandlungen immer noch das Bild prägen, führten in den USA Dezentralisierungsprozesse zur weitgehenden Ablösung kollektiver Standards durch einzelvertragliche Regelungen.

\section{Fragestellung und Methode}

In Deutschland hingegen vollzog sich Dezentralisierung nicht auf dem Wege eines radikalen Bruchs mit formalen Tarifstrukturen, sondern durch deren Anpassung und Öffnung in Richtung größerer betrieblicher Gestaltungsmöglichkeiten. Diese Form der Dezentralisierung vollzog sich in mehreren Etappen und nahm ihren Ausgangspunkt in der Auseinandersetzung um die Einführung der 35-Stunden Woche. ${ }^{2}$ Die Besonderheit des deutschen Falles liegt nicht nur in der inkrementellen Einführung von Dezentralisierung, sondern darin begründet, dass hier neben den Beschäftigten, Gewerkschaften und Arbeitgebern (bzw. Arbeitgeberverbänden) auch Be-

\footnotetext{
1 Der Verfasser dankt Wolfram Brehmer, Markus Helfen, Werner Nienhüser, Britta Rehder sowie den Teilnehmerinnen und Teilnehmern des AIAS/ WSI Workshops "Neue Perspektiven betrieblicher Interessenvertretung" für wichtige Anregungen und Kommentare.

2 Den Einstieg in die Arbeitszeitverkürzung erkaufte sich die IG Metall im Jahr 1984 durch eine betriebliche Flexibilisierung der Arbeitszeitregime, welche letztlich auf dem Wege der Öffnung des Flächentarifvertrages zustande kam (Thelen 1991; Schmidt/Trinczek 1986).
}

Martin Behrens, Dr. (USA), Wissenschaftler im WSI in der Hans-Böckler-Stiftung. Arbeitsschwerpunkte: Komparative industrielle Beziehungen, Arbeitsbeziehungen in den USA, Arbeitgeberverbände, Tarifpolitik. e-mail: martin-behrens@boeckler.de 
triebsräte als wesentliche Akteure ins Spiel kommen. Während zahlreiche Arbeiten die Verbetrieblichungsneigung der betrieblichen Akteure selbst analysieren (Bispinck/ Schulten 2003; Nienhüser/Hoßfeld 2007) und die Konsequenzen von Dezentralisierung für die Tarifabschlüsse thematisiert haben (Bispinck 2004; Schnabel 2006; Traxler 2003; Schroeder/Weinert 1999), waren deren Auswirkungen auf die Arbeit der Betriebsräte selbst vergleichsweise selten Gegenstand wissenschaftlicher Erörterungen.

Im Folgenden soll nun mit Rückgriff auf die Daten der vierten WSI-Befragung von Betriebs- und Personalräten (2004/05) der Frage nachgegangen werden, inwieweit sich unterschiedliche Formen der Dezentralisierung auf die grundlegenden Handlungsmöglichkeiten der Betriebsräte auswirken. Die Analyse basiert auf der telefonischen Befragung (CATI) von 2.000 Betriebsräten sowie 1.300 Personalräten, die vom Institut für angewandte Sozialwissenschaften (infas) im Auftrag des Wirtschaftsund sozialwissenschaftlichen Instituts in der Hans-Böckler-Stiftung (WSI) durchgeführt wurde. Die Interviews wurden im Frühjahr 2005 zumeist mit den jeweiligen Betriebsrats- bzw. Personalratsvorsitzenden geführt. Für die nun folgenden Auswertungen wurde lediglich die Betriebsrätebefragung zugrunde gelegt. Die Auswahl der Interviews erfolgte auf der Basis einer disproportionalen Zufallsstichprobe von 13.760 Adressen aus der Betriebsdatei der Bundesagentur für Arbeit (BA). Für die Befragung wurden nur Betriebsstätten aus der privaten Wirtschaft berücksichtigt, die mehr als 20 Beschäftigte aufweisen. Die Ausschöpfung der Stichprobe betrug nach Abzug der neutralen Ausfälle (z. B. kein Betriebsrat existent, fehlende Telefonnummer) 66,4 \% (Betriebs- und Personalräte).

Die nun folgende Analyse klammert Dezentralisierungsfolgen im Bereich der Tarifpolitik aus und konzentriert sich auf die bislang wenig untersuchten Konsequenzen für die betriebliche Mitbestimmung. Dabei stehen folgende Leitfragen im Vordergrund: Führt eine zunehmende Dezentralisierung tarifpolitischer Entscheidungen im Sinne einer „Verbetrieblichung“ zu einer Überforderung betrieblicher Akteure, oder, wie Artus (2003, S. 252f.; 2004, S. 275) es mit Blick auf die Verhältnisse in Ostdeutschland ausdrückt, letztlich zur „Destabilisierung" beider Säulen der industriellen Beziehungen? Oder ist die eingeschränkte Fähigkeit deutscher Arbeitsbe- ziehungen zur Anpassung an veränderte Rahmenbedingungen bereits in der Struktur des dualen Systems selbst, und hierbei insbesondere in der fehlenden betrieblichen Verankerung der Gewerkschaften, angelegt, wie Ilsøe et al. (2007, S. 217f.) behaupten? Führt Dezentralisierung in Gestalt konzessionärer (betrieblicher) „Bündnisse für Arbeit" zur Aufzehrung betrieblicher Tauschpotenziale (Massa-Wirth/Seifert 2004, S. 254; Massa-Wirth 2007, S. 214ff.) und somit in langer Frist zur Erschöpfung von Legitimitätsressourcen der betrieblichen Mitbestimmung (Rehder 2006, S. 228f.)?

Wie bisherige Befunde andeuten, erscheint es nur schwer vorstellbar, dass Legitimitätserschöpfung (Rehder), Systeminsuffizienz (Ilsøe et al.) oder Systemdestabilisierung (Artus) die Effektivität der Betriebsratsarbeit gänzlich unberührt lassen. Wie weitgehend nun diese Effekte sind und inwieweit sich unterschiedliche Formen der Dezentralisierung identifizieren lassen, die dann möglicherweise auch unterschiedlichen Einfluss auf die Effektivität der Betriebsratsarbeit ausüben, wird im Folgenden genauer untersucht. Hierbei folgt die weitere Analyse der Leithypothese, dass sich die Folgen der Dezentralisierung von Tarifpolitik für die Effektivität der Betriebsratsarbeit nur dann sinnvoll bestimmen lassen, wenn Dezentralisierung auf ihre Ursprungs- und Begründungszusammenhänge zurückgeführt und somit disaggregiert wird. Wie die eingangs präsentierten Befunde aus der komparativen Forschung deutlich gemacht haben, kann nämlich Dezentralisierung je nach Ausgangspunkt des Prozesses und je nach spezifischer Einbettung in die nationalen Systeme der Arbeitsbeziehungen unterschiedliche Bedeutungen annehmen. Der Zentralisierungsbegriff muss also zunächst „entschlüsselt“ werden, bevor er sinnvoll im Rahmen einer empirischen Analyse verwendet werden kann.

In Abschnitt 3 werden daher zunächst die unterschiedlichen Erscheinungsformen von Dezentralisierung analysiert und systematisiert. In Abschnitt 4 wird die Verbreitung unterschiedlicher Dezentralisierungsformen in deutschen Betrieben dargestellt und anschließend einer multivariaten statistischen Analyse unterzogen. Im Abschnitt 5 werden die Ergebnisse diskutiert.

\section{Formen der Dezentralisierung}

Dezentralisierung vollzog sich in unterschiedlichen Geschwindigkeiten, aber auch in unterschiedlichen Ausprägungen. So lässt sich nach Traxler (1995) unter „organized decentralization"3 eine Form der Dezentralisierung verstehen, welche zwar die Aufgabe der Tarifverhandlungen auf Akteure einer unteren Verhandlungsebene (lower-level associations) verlagert, jedoch eine wirkungsvolle Kontrolle dieser Verhandlungen durch Kollektivakteure auf höherer Ebene beibehält. Tarifpolitik erweist sich bei dieser Dezentralisierungsform zwar nicht mehr als zentralisiert, aber immer noch als koordiniert. Nach Traxler (1995, S. 7) kam es zur Ablösung offener Formen der Koordinierung durch die Koordinierungsfunktion der Spitzenorganisationen der Arbeitgeber und Gewerkschaften bzw. durch die Verhandlungsführerschaft bestimmter Verbände. Von diesem Muster lässt sich „disorganized decentralization" als eine Form reiner Deregulierung von Tarifbeziehungen unterscheiden. ${ }^{4}$

Während Traxlers Ursprungskonzept noch sehr stark vom Resultat der Dezentralisierung her gedacht und in eine international vergleichende Forschungsperspektive eingebettet war, stellten einige der darauf folgenden Konzeptionalisierungen für den deutschen Kontext stärker unterschiedliche Dezentralisierungsverfahren in den Mittelpunkt. Anknüpfend an Zacherts Begrifflichkeit (Zachert 1997, S. 413) unterscheiden Bispinck/Schulten (1999) zwischen „kontrollierter“ und „wilder“ Dezentralisierung. Demnach lassen sich unter kontrollierter Dezentralisierung solche Formen zusammenfassen, welche direkt Regelungskompetenzen an die Betriebsparteien übertragen oder ,auf überbetrieblicher Ebene bestimmte Konditionen definieren,

3 Unter dem Begriff "centrally coordinated decentralization " wird dieser Aspekt bereits bei Ferner/ Hyman angedeutet (1992, S. xxxvi), allerdings nicht systematisch weiterentwickelt.

$4 \quad$ Als Prototyp dieser Form verweist Traxler auf das Beispiel Neuseeland. Hier wurde im Zuge der Arbeitsrechtsreform des Jahres 1991 überbetrieblichen Tarifbeziehungen der Boden weitgehend entzogen. 
unter denen die betrieblichen Akteure von den betrieblichen Standards abweichen dürfen“" (Bispinck/Schulten 1999, S. 198). Als bevorzugtes Instrument einer solchen kontrollierten Dezentralisierung erweist sich die tarifliche Öffnungsklausel. Weist noch bei Zachert der Begriff der „wilden“ Dezentralisierung einen sehr engen Bezug zur Rechtswidrigkeit des Arbeitgeberhandels auf, so wird diese Form bei Bispinck/ Schulten weiter gefasst. Neben faktischem Tarifbruch durch eine vom Tarifvertrag (rechtswidrig) abweichende betriebliche Praxis kommt hier auch der Verbandsaustritt von Arbeitgeberverbandsmitgliedern bzw. die Verweigerung des Eintritts durch Verbandsaußenseiter ins Spiel (Bispinck/ Schulten 1999, S. 201f.). ${ }^{5}$

An diese Begrifflichkeit soll hier angeknüpft werden, jedoch gilt es mit Bezug auf die betrieblichen Arbeitsbeziehungen einige Präzisierungen vorzunehmen. So erweist sich „wilde Dezentralisierung“ (Bispinck/Schulten in Anknüpfung an $\mathrm{Za}$ chert) bzw. „Desorganisation“ (Schroeder/ Weinert) als nicht hinreichend trennscharf, weil zumindest im Falle der Verbandsabstinenz eine informelle Koordination, z. B. durch eine freiwillige Orientierung der Unternehmen an den Vorschriften des Tarifvertrages, nicht ausgeschlossen werden kann. Auch verhindern im Fall des Verbandsaustritts die tarifvertragsrechtlichen Vorschriften der Nachbindung (bis zum Ablauf eines Tarifvertrages) und der Nachwirkung (nach Ablauf) eine vollständige oder weitgehende Deregulierung der Tarifbeziehungen, sodass in vielen Fällen das Echo von Koordination noch Jahre nach Verbandsaustritt zu vernehmen ist. Gerade aber für die Arbeit des Betriebsrates ist die faktische Geltung von Tarifvorschriften und nicht allein die kollektivvertragliche Tarifbindung entscheidend, denn Erstere definiert überhaupt erst die Notwendigkeit, die betrieblichen Interessenvertreter als subsidiären Tarifakteur ins Spiel zu bringen. Für den Zweck der hier vorzunehmenden Analyse sollen daher unter dem Begriff der „unkontrollierten“ oder „wilden" Dezentralisierung ausschließlich solche Formen der Abweichung von bestehenden Verbandstarifverträgen verstanden werden, die im Konflikt mit geltenden Tarifbestimmungen zustande kommen (unabhängig davon, auf welcher Rechtsgrundlage diese Geltung beruht). Unter „kontrollierter" Dezentralisierung werden anschlieBend an etablierte Dezentralisierungskon- zepte solche Formen gefasst, die auf einer zwischen Gewerkschaften und Arbeitgeberverbänden ausgehandelten Öffnungsklausel basieren.

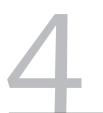

\section{Einfluss von Dezentralisierung auf Betriebsratsarbeit}

Bevor nun der Einfluss von kontrollierter und unkontrollierter Dezentralisierung auf die Arbeit des Betriebsrates näher analysiert wird, gilt es hier zunächst eine Verständigung darüber herbeizuführen, was genau unter „Effektivität" der Betriebsratsarbeit zu verstehen und wie diese zu operationalisieren ist.

\subsection{KANN MAN DIE EFFEKTIVITÄT DER BETRIEBSRATSARBEIT BESTIMMEN?}

Eine erste Möglichkeit der Bestimmung von Effektivität würde von den rechtlichen Grundlagen der Betriebsratsarbeit ausgehen. Demnach wäre die Effektivität der Betriebsratspraxis an den Intentionen der Betriebsverfassung, also dem Betriebsverfassungsgesetz als Konstitutionsgesetz des Betriebsrats (BR), zu messen. Wie Rosenbaum (1982, S. 398) herausgearbeitet hat, würde dies bereits regelmäßig daran scheitern, dass der Verabschiedung des Gesetzes im Jahre 1952 sowie den darauf folgenden Gesetzesreformen unterschiedliche, teils offen konfligierende Intentionen zugrunde lagen, die sich dann auch nur in ihrer Widersprüchlichkeit rekonstruieren ließen. Die Bestimmung der Effektivität der Betriebsratsarbeit in Relation zu den Intentionen des Gesetzgebers scheidet daher aus.

Eine zweite Möglichkeit bestünde darin, statt der Intentionen des Gesetzgebers den Vollzug des Gesetzes, gemessen an der Einhaltung der Gesetzesnormen, als Bewertungsmaßstab heranzuziehen. Folgt man weiten Teilen der Betriebsräteforschung, so hat sich mittlerweile in vielen Bereichen eine vom Gesetz abweichende Praxis etabliert, welche - oftmals auf dem Wege komplexer Tauschgeschäfte - den spezifischen Interessen der betrieblichen Akteure auf dem Verhandlungswege folgt, dem Gesetz jedoch nicht vollständig Rechnung trägt (Weltz 1977; Rosenbaum 1982;
Rehder 2003; Seifert/Massa-Wirth 2005). Letztlich würde also die Bestimmung der Vertretungseffektivität verstanden als reine Erforschung des Gesetzesvollzugs diese dynamische Fortentwicklung der Betriebsratspraxis aus den Augen verlieren.

Schließlich bietet sich drittens die Betriebsratsperspektive als Bewertungsmaßstab an. Da weite Teile der Betriebsverfassung als Grundlagen zur kollektiven Partizipation der Beschäftigten gesehen werden können, und mithin der Betriebsrat als der gewählte Repräsentant, könnte die Effektivität der Betriebsratsarbeit auch an den subjektiven Erwartungen des Partizipationserfolgs gemessen werden. In einer solchen Betriebsratsperspektive misst sich Effektivität nicht ausschließlich an der Befolgung von Gesetzen, sondern an den Partizipationserwartungen der Interessenvertreter. In diese Partizipationserwartungen gehen neben den rechtlich kodierten Beteiligungsmöglichkeiten auch die betrieblichen Ausgangsbedingungen, individuellen Orientierungen und die an den Betriebsrat kommunizierten Beteiligungswünsche der Belegschaften ein. An diese Perspektive soll im Weiteren angeknüpft werden.

Zur Bestimmung dieser subjektiven Bewertungen der Effektivität von Betriebsratsarbeit wird auf einen besonderen Fragenblock der vierten WSI-Befragung von Betriebs- und Personalräten (2004/05) zurückgegriffen. ${ }^{6}$ Betriebsräte wurden in dieser Befragung nicht nur um Auskunft darüber gebeten, mit welchen Themen sie sich während der vorangegangenen zwei Jahre beschäftigt haben, sondern auch darüber, wie sie im Bereich der von ihnen genannten Themen den Erfolg ihrer Arbeit bewerten (eher viel erreicht/eher wenig erreicht). Insgesamt wurden den befragten Betriebsräten 36 Themen vorgelegt, die aus vorangegangenen Befragungen und zusätzlich im Pre-Test genannten Kategorien gewonnen wurden.

\footnotetext{
5 Verwandte Konzeptionalisierungen finden sich unter der Terminologie „Dezentralisierung vs. Desorganisation " bei Schroeder/Weinert (1999) und bei Artus (2003, S. 251) unter der Begrifflichkeit äußere Erosion vs. innere Erosion “.

6 Spätere Betriebsrätebefragungen des WSI (WSIBetriebsrätebefragung zur Restrukturierung 2006; Fünfte WSI-Befragung von Betriebsräten 2007) enthalten diesen Fragenblock nicht mehr bzw. nicht in der erforderlichen Ausdifferenzierung, sodass für die vorliegende Analyse auf eine frühere Befragung zurückgegriffen wurde.
} 
Tabelle 1: Themen der Betriebsratsarbeit "Eher viel erreicht" - in \% -

\begin{tabular}{|c|c|c|c|c|c|}
\hline & $\begin{array}{l}\text { Ohne „wilde" } \\
\text { Dezentrali- } \\
\text { sierung }\end{array}$ & $\begin{array}{l}\text { Mit „wilder“ } \\
\text { Dezentrali- } \\
\text { sierung }\end{array}$ & $\begin{array}{c}\text { Ohne } \\
\text { koordinierte } \\
\text { Dezentrali- } \\
\text { sierung }\end{array}$ & $\begin{array}{c}\text { Mit } \\
\text { koordinierter } \\
\text { Dezentrali- } \\
\text { sierung }\end{array}$ & Fallzahl \\
\hline $\begin{array}{l}\text { Sozialplan/Interessen- } \\
\text { ausgleich }\end{array}$ & 84,0 & 77,3 & 85,4 & 82,4 & 537 \\
\hline $\begin{array}{l}\text { Familienfreundliche } \\
\text { Arbeitsbedingungen }\end{array}$ & 57,1 & 41,7 & 51,5 & 57,1 & 629 \\
\hline Untertarifliche Entlohnung & 71,3 & 41,7 & 62,8 & 63,8 & 231 \\
\hline Einführung neuer Techniken & 76,7 & 70,2 & 76,4 & 76,0 & 875 \\
\hline Überstunden & 53,0 & 48,8 & 51,6 & 52,8 & 754 \\
\hline Fort- und Weiterbildung & 69,4 & 57,7 & 66,3 & 69,0 & 1068 \\
\hline
\end{tabular}

Aus diesen 36 thematisch sehr weit streuenden Kategorien wurden für die weitere Analyse sechs Themen identifiziert, die näherungsweise die Komplexität der Betriebsratsarbeit abbilden. Die Identifikation dieser Themen erfolgte unter Zuhilfenahme einer Faktorenanalyse (Hauptkomponentenanalyse mit Varimax Rotation) über die zunächst die hinter den 36 Themen stehenden latenten Variablen bestimmt wurden. Für die weitere vertiefende Analyse wurden dann innerhalb der Faktoren jene Themenfelder verwendet, die jeweils die höchste Faktorenladung aufweisen. Diese Themen sind: Sozialplan/ Interessenausgleich, familienfreundliche Arbeitsbedingungen, untertarifliche Entlohnung, Einführung neuer Techniken, Einführung neuer Arbeitszeitformen, sowie Fort- und Weiterbildung.

\subsection{DIE WIRKUNGEN UNTERSCHIEDLICHER DEZENTRALISIERUNGSFORMEN}

Für die insgesamt sechs ausgewählten Themenbereiche wird in der Folge analysiert, wie die Betriebsräte den Erfolg ihrer Arbeit bewerten. Die Antworten („haben eher viel erreicht") wurden nach den beiden unterschiedlichen Dezentralisierungsformen differenziert.

Wie die Anteilswerte in Tabelle 1 zeigen (Spalten 1 und 2) wird der eigene Erfolg der Betriebsratsarbeit in allen Themenfeldern vergleichsweise negativ bewertet, wenn es im Betrieb zur wilden Dezentralisierung gekommen ist. Diese durchgängig vorliegende Korrelation zwischen wilder Dezentralisierung und schlechter Bewertung des eigenen Erfolgs ist umso bemerkenswerter, da nicht alle der präsentierten Themenfelder in einem unmittelbaren Zusammenhang mit dem Tarifgeschehen stehen. Es deutet sich an, dass wilde Dezentralisierung neben den direkten Auswirkungen auf Vergütung und Arbeitszeitregime auch auf die Bewertung von solchen Themenfeldern ausstrahlt, die nicht unmittelbar mit dem Tarifgeschehen im Zusammenhang stehen. Möglicherweise beeinträchtigt wilde Dezentralisierung das Verhältnis zwischen Management und Betriebsrat insofern, dass der Betriebsrat auch in diesen (nicht tariflich geregelten) Arbeitsfeldern seltener zu für ihn befriedigenden Lösungen kommt.

Betrachtet man nun den Zusammenhang zwischen Bewertung des Erfolgs und der kontrollierten Dezentralisierung, so zeigen sich keine vergleichbaren Effekte. Wie die Prozentangaben (Spalten 3 und 4 von Tabelle 1) andeuten, schlägt sich das Vorliegen von kontrollierter Dezentralisierung nicht per se in einer schlechteren Bewertung des eigenen Erfolgs nieder. In einer Reihe von Themenfeldern, wie z. B. den „Familienfreundlichen Arbeitsbedingungen“ und „Fort- und Weiterbildung“, scheint koordinierte Dezentralisierung positiv mit der Einschätzung des Erfolgs verbunden zu sein. Nun ist an dieser Stelle Vorsicht angebracht, denn die präsentierten Daten könnten durch bislang nicht berücksichtigte Einflüsse, z. B. der Betriebsgröße und Branche, verzerrt sein. Um an dieser Stelle mehr Gewissheit zu erlangen, werden in der Folge für einzelne Themenfelder getrennte Schätzungen durchgeführt, die neben den beiden hier interessierenden Dezentralisierungsformen eine Reihe weiterer Einflussgrößen berücksichtigen.

\subsection{MULTIVARIATE SCHÄTZMODELLE}

Insgesamt werden jeweils nur solche Fälle in die Schätzungen einbezogen, die eine Bindung an einen Flächentarifvertrag aufweisen. Dies erscheint notwendig, da beide
Dezentralisierungsformen überhaupt nur Sinn ergeben, wenn der Flächentarifvertrag als Referenzpunkt besteht. Weiterhin werden in den einzelnen Schätzmodellen nur solche Betriebe berücksichtigt, in denen sich der Betriebsrat mit dem genannten Thema beschäftigt hat, schließlich ist der Erfolg (oder der Misserfolg) davon abhängig, ob das Thema für den jeweiligen Betriebsrat überhaupt relevant ist. Die beiden genannten Restriktionen führen in einigen Fällen zu stark verringerten Gesamtfallzahlen, ein Umstand, der eine sparsame Modellierung der Schätzmodelle gebietet.

Zur Bestimmung der Einflussfaktoren der Betriebsratseffektivität werden zunächst die eingangs definierten Formen der Dezentralisierung in ein multivariates Schätzmodell einbezogen. Wilde/unkontrollierte Dezentralisierung wird hier durch jene Fälle repräsentiert, in denen Betriebsräte angaben, zur Abweichung von bestehenden Tarifvorschriften nach unten gedrängt worden zu sein. ${ }^{7}$ Insgesamt 10,6 \% der befragten Betriebsräte in Betrieben mit Verbandstarifbindung gaben an, dass es in ihrem Betrieb zur unkoordinierten Dezentralisierung gekommen ist, wobei davon auszugehen ist, dass dieser Wert wegen der sozialen Unerwünschtheit des Tatbestandes der Tarifabweichung das wirkliche Ausmaß dieser Dezentralisierungsform eher unterschätzt. Die Variable „kontrollierte Dezentralisierung" kennzeichnet hingegen jene Fälle, in denen nach Aussage des Betriebsrates mindestens eine Öffnungsklausel genutzt wird. 72,9\% der Betriebsräte (nur Betriebe mit Verbandstarif) gaben an, Öffnungsklauseln in Anspruch zu nehmen, wobei die Wahrscheinlichkeit der Nutzung mit der Betriebsgröße ansteigt. Die in die Analyse einbezogenen Dezentralisierungsvariablen schließen einander nicht aus. So nutzt eine Vielzahl von Betrieben sowohl Öffnungsklauseln als auch darüber hinausweisende „wilde“ Abweichungen vom Tarifvertrag.

Neben den beiden Dezentralisierungsvariablen kontrolliert das präsentierte Schätzmodell eine Reihe von Rahmenbedingungen der Betriebsratsarbeit sowie Charakteristika des Betriebs. So gibt die Va-

\footnotetext{
Der Wortlaut der Frage war: „In vielen Betrieben drängen die Arbeitgeber auf Unterschreitung tariflicher Standards, um die Kosten- und Wettbewerbssituation zu verbessern. Gibt es in Ihrem Betrieb Abweichungen von bestehenden Tarifvorschriften nach unten?"
} 
riable „Gesamtbetriebsrat" an, ob ein für den örtlichen Betriebsrat zuständiger Gesamtbetriebsrat existiert. Es ist davon auszugehen, dass mit der Einbettung des Betriebsrats in eine Multiebenenstruktur der Betriebsratsarbeit zusätzliche Ressourcen aktiviert werden können, die sich positiv auf die Arbeit des Gremiums auswirken (Behrens/Kädtler 2008). Ein ähnlicher Unterstützungseffekt ist bezogen auf den gewerkschaftlichen Organisationsgrad des Betriebsratsgremiums anzunehmen. Demnach kann ein vergleichsweise gut gewerkschaftlich angebundener Betriebsrat Unterstützungs- und Beratungsdienstleistungen der Gewerkschaft mobilisieren, welche sich dann wiederum positiv auf die Effektivität der Betriebsratsarbeit auswirken dürften. Dies gilt allerdings mit einer Einschränkung: Letztlich könnten durch die enge Bindung an die Gewerkschaft auch zusätzliche Erwartungen von außen an den Betriebsrat herangetragen werden, was dann die Erwartungen an "gute Betriebsratsarbeit" erhöht. Da bei der hier verwendeten Messung von Betriebsratseffektivität über die Selbsteinschätzung der befragten Betriebsräte Erwartungen eine wichtige Rolle spielen, könnte es sein, dass somit der positive Unterstützungseffekt quasi neutralisiert oder gar überkompensiert wird.

Eine weitere Variable nimmt den Wert 1 an, wenn es innerhalb des Betriebes während der letzten zwei Jahre zum Personalabbau gekommen ist. Somit berücksichtigt diese Variable ökonomische Krisensituationen, die potenziell neue Problemkonstellationen für die Betriebsratsarbeit schaffen und die Interessenvertretung unter verstärkten Handlungsdruck setzen. Weitere Variablen kontrollieren die Branchenzugehörigkeit sowie die Betriebsgröße (logarithmierte Zahl der Beschäftigten). Die Häufigkeiten und Mittelwerte (ungewichtete Daten) der genannten Variablen finden sich in Tabelle 2.

\subsection{ERGEBNISSE DER SCHÄTZUNG}

Insgesamt lassen die sechs geschätzten Modelle schon allein wegen der stark differierenden Fallzahlen keinen Vergleich der Effekte bei den unterschiedlichen Dezentralisierungsformen zwischen den verschiedenen Themenfeldern zu. Sie verweisen aber darauf, ob die beobachteten Differenzen bei der Selbsteinschätzung des Betriebsratserfolgs auch bei statistischer Kontrolle entscheidender Schlüsselvariablen wie Be-

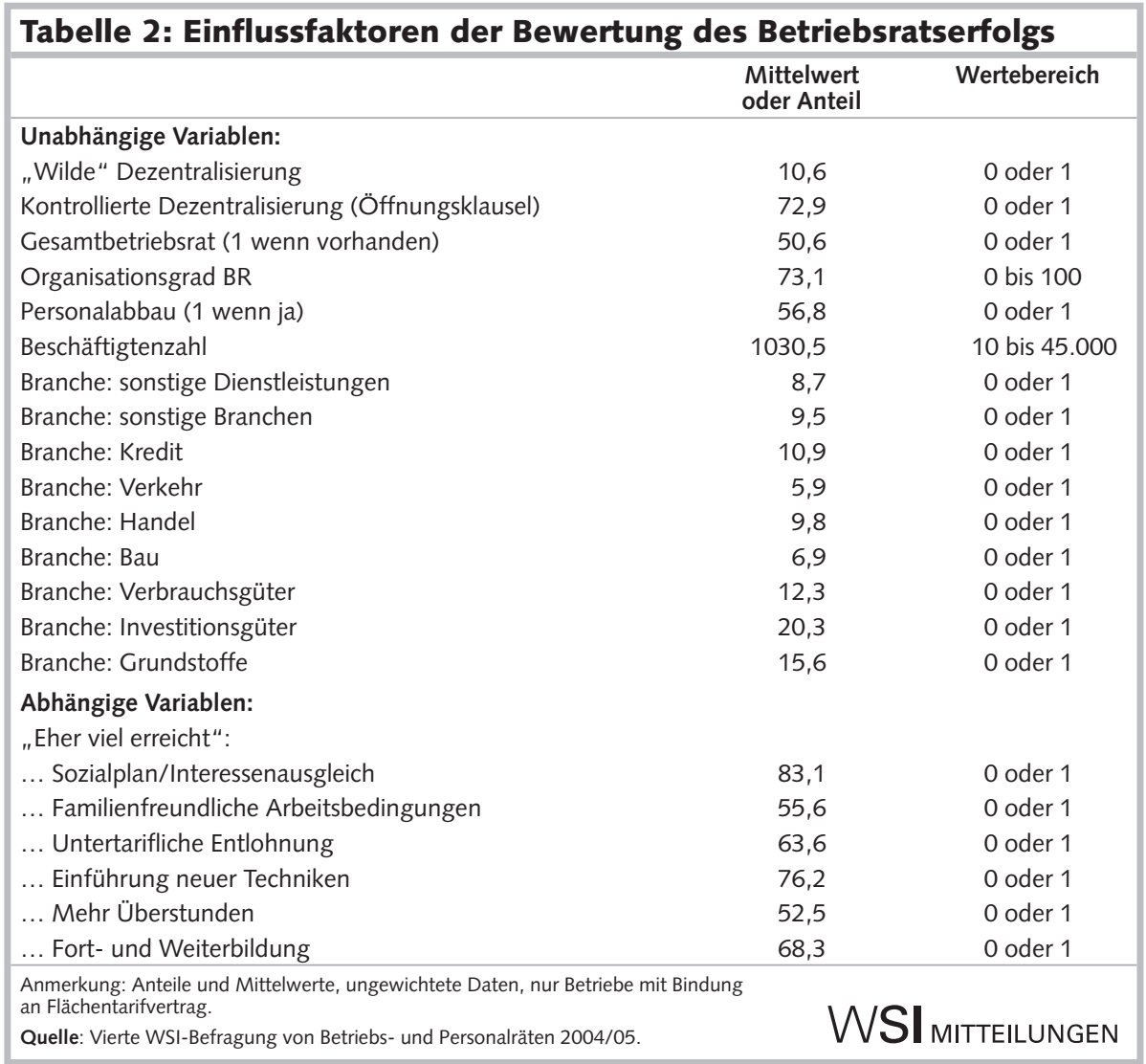

triebsgröße und Branche Bestand haben. Insgesamt erweist sich der Erklärungsbeitrag von fünf der sechs Modelle als recht gering, was wohl im Wesentlichen auf zwei Gründe zurückzuführen sein dürfte: Erstens sprechen die genannten Themenbereiche unterschiedliche Aspekte der betrieblichen Realität an, die bei Anwendung eines einheitlichen Grundmodells der erklärenden Variablen letztlich nicht jeweils innerhalb ihres spezifischen betrieblichen Kontextes abgebildet werden können. Zweitens sind subjektive Einschätzungen von Erfolg oder Misserfolg recht stark von individuellen Persönlichkeitsmerkmalen und erwerbsbiografischen Aspekten abhängig, die im Rahmen der hier vorliegenden Betriebsrätebefragung nicht erhoben wurden.

Trotz der genannten Restriktionen der Modelle, die eine zurückhaltende Interpretation nahelegen, deutet die vorgelegte Analyse zunächst einige Zusammenhänge zwischen wahrgenommenem Erfolg der Betriebsratsarbeit und unterschiedlichen Formen der Dezentralisierung an, die über den Vergleich der Häufigkeiten hinausgehen. Diese sollen nun in summarischer Form präsentiert werden.

Zunächst einmal trägt die Variable „wilde Dezentralisierung“ in allen sechs
Schätzungen das erwartete negative Vorzeichen (Tabelle 3). Signifikante Koeffizienten ergeben sich bei der Bewertung des Betriebsratserfolges bei untertariflicher Entlohnung (signifikant auf dem $1 \%-\mathrm{Ni}$ veau), der familienfreundlichen Arbeitsbedingungen (5\%) sowie der Fort- und Weiterbildung (5\%). Während es wohl kaum überrascht, dass die Abweichung vom Flächentarifvertrag mit der schlechten Bewertung des Betriebsratseinflusses im Themenbereich „untertarifliche Entlohnung“ in Verbindung steht, erweisen sich die negativen Effekte bei Fort- und Weiterbildung sowie familienfreundlichen Arbeitsbedingungen als erklärungsbedürftig. Bei beiden Themen handelt es sich um tarifferne Aspekte, die (zumindest im Jahr der Befragung) recht selten durch Tarifverträge geregelt wurden. ${ }^{8} \mathrm{Da}$ in beiden Fällen, anders als beim Sozialplan/Interessenausgleich und den Überstunden, auch keine starke Beteiligungsregelung des Betriebsverfassungsgesetzes vorliegt, die quasi als untere Auffanglinie auch in harten Zeiten

8 Zum Zeitpunkt der Erhebung (2004/05) hatte die IG Metall zwar einen Qualifizierungstarifvertrag ausgehandelt, dieser galt aber zunächst (bis 2006) nur für die Tarifgebiete des Landes Baden-Württemberg. 
Tabelle 3: Binär logistische Regressionen: Erfolg der Betriebsratsarbeit in verschiedenen Themenfeldern (Koeffizienten $B$ )

\begin{tabular}{|c|c|c|c|c|c|c|}
\hline & $\begin{array}{c}\text { Modell } 1 \\
\text { Sozialplan/ } \\
\text { Interessen- } \\
\text { ausgleich }\end{array}$ & $\begin{array}{l}\text { Modell } 2 \\
\text { Familien- } \\
\text { freundliche } \\
\text { Arbeitsbe- } \\
\text { dingungen }\end{array}$ & $\begin{array}{c}\text { Modell } 3 \\
\text { Unter- } \\
\text { tarifliche } \\
\text { Entlohnung }\end{array}$ & $\begin{array}{c}\text { Modell } 4 \\
\text { Einführung } \\
\text { neuer } \\
\text { Techniken }\end{array}$ & $\begin{array}{l}\text { Modell } 5 \\
\text { Über- } \\
\text { stunden }\end{array}$ & $\begin{array}{l}\text { Modell } 6 \\
\text { Fort- und } \\
\text { Weiter- } \\
\text { bildung }\end{array}$ \\
\hline $\begin{array}{l}\text { "Wilde" } \\
\text { Dezentralisierung }\end{array}$ &,- 306 &,$- 622 * *$ & $-1,275^{* * *}$ &,- 237 &,- 119 &,$- 446 * *$ \\
\hline $\begin{array}{l}\text { Kontrollierte } \\
\text { Dezentralisierung }\end{array}$ &,- 164 & 273 & ,200 & ,031 &,- 022 & 192 \\
\hline $\begin{array}{l}\text { Gesamtbetriebsrat } \\
\text { (1 wenn vorhanden) }\end{array}$ &, $631^{* *}$ & 116 & ,267 & ,099 & ,082 &, $442 * * *$ \\
\hline Organisationsgrad BR &,- 003 &,- 004 & ,003 &,- 002 & 002 & ,004 \\
\hline $\begin{array}{l}\text { Personalabbau } \\
\text { (1 wenn ja) }\end{array}$ & ,371 &,- 058 & 479 &,$- 583 * * *$ &,- 170 &,- 155 \\
\hline Beschäftigtenzahl (log.) &, $275^{* *}$ & ,003 &, $281 *$ &, $207 * * *$ &,$- 183 * *$ &, $169 * *$ \\
\hline \multicolumn{7}{|l|}{$\begin{array}{l}\text { Branche: sonstige } \\
\text { Dienstleistungen } \\
\text { (Referenz) }\end{array}$} \\
\hline $\begin{array}{l}\text { Branche: sonstige } \\
\text { Branchen }\end{array}$ &,- 221 & $1,235^{* * *}$ &,- 912 & 461 & 097 &, $984 * * *$ \\
\hline Branche: Kredit &,- 516 & 148 &, 585 & , 145 & $630^{*}$ &, 464 \\
\hline Branche: Verkehr &,- 981 &,- 303 & 109 & $1,093 * *$ & $1,080 * *$ & 187 \\
\hline Branche: Handel & $-1,628 * *$ &,- 001 & ,639 &, 409 &, $635^{*}$ &,- 036 \\
\hline Branche: Bau & $-1,164^{*}$ &,- 013 &, 435 & 274 & 112 & 167 \\
\hline $\begin{array}{l}\text { Branche: Verbrauchs- } \\
\text { güter }\end{array}$ &,- 565 &,- 011 & ,257 &, $697^{*}$ & $838 * *$ &,- 320 \\
\hline $\begin{array}{l}\text { Branche: Investitions- } \\
\text { güter }\end{array}$ &,- 452 & 012 & ,672 &,- 139 & $1,110 * * *$ &,- 373 \\
\hline Branche: Grundstoffe &,- 158 & ,256 & 211 &,- 123 & 479 &,- 289 \\
\hline Konstante &, 254 & 207 & $-1,990^{*}$ & 210 &, 536 & $-0,798$ \\
\hline $\begin{array}{l}\text { Zahl der gültigen Fälle } \\
\text { Nagelkerke } \mathrm{R}^{2}\end{array}$ & $\begin{array}{r}522 \\
0,100\end{array}$ & $\begin{array}{r}616 \\
, 055\end{array}$ & $\begin{array}{r}227 \\
0,197\end{array}$ & $\begin{array}{r}856 \\
0,056\end{array}$ & $\begin{array}{r}738 \\
0,062\end{array}$ & $\begin{array}{r}1040 \\
0,067\end{array}$ \\
\hline
\end{tabular}

dienen könnte, drängt sich hier die Annahme auf, es könnte sich um Ausstrahlungseffekte handeln. Demnach trägt wilde Dezentralisierung zur Zerrüttung des Kooperationsverhältnisses zwischen Management und Betriebsrat bei, was sich dann in einer negativen Bewertung des Betriebsratserfolges ausdrückt. Zumindest aus der hier untersuchten Betriebsratsperspektive erscheint die Geschäftsgrundlage für eine weitere vertrauensvolle Zusammenarbeit als entzogen.

Betrachtet man nun die Koeffizienten für kontrollierte Dezentralisierung, so lässt sich hier kein Hinweis auf ähnlich lähmende Effekte finden. Koeffizienten tragen unterschiedliche Vorzeichen und sind in keinem Fall signifikant. Möglicherweise würde eine weitere Differenzierung der Inhalte von Öffnungsklauseln weiteren Aufschluss liefern. Eine solche vertiefende Analyse ist allerdings wegen der geringen Fallzahlen nicht möglich. Weiterhin bleibt zu berichten, dass die Existenz eines Gesamtbetriebsrates in zwei Fällen (Fort- und Weiterbildung und Sozialplan/Interessenausgleich) positiv mit dem eingeschätzten Erfolg assoziiert ist, der Einfluss des ge-
Wir wissen aus mehreren Untersuchungen, dass Betriebsräte, wenn sie die Wahl hätten, mit großer Mehrheit auf eine Dezentralisierung der Tarifpolitik verzichten würden. Fragt man nämlich Betriebsräte direkt nach ihren Einschätzungen und Wünschen in Bezug auf Dezentralisierung, so kann Bispinck mit Bezug auf die Daten der auch hier verwendeten Befragung zeigen, dass eine Mehrheit von $53 \%$ eine ,Verbetrieblichung“ der Tarifpolitik "generell problematisch" findet und weitere $30 \%$ dieser „Zwiespältig“ gegenüberstehen (Bispinck 2005, S. 304f.). Auch stimmen $81 \%$ der befragten Interessenvertreter der Aussage zu, dass Dezentralisierung dem Arbeitgeber eher die Möglichkeit gibt, seine betrieblichen Interessen durchzusetzen (ebd.). Ähnliche Befunde finden sich auch in der Untersuchung von Nienhüser/Hoßfeld. Gefragt nach den erwarteten Folgen einer stärkeren Abkehr vom Flächentarifvertrag geben $78 \%$ der Betriebsräte zu Protokoll, dies würde zu mehr Konflikten im Betrieb führen, und $79 \%$ verbinden mit einer solchen Entwicklung sinkende Löhne (Nienhüser/Hoßfeld 2007, S. 301), wobei aus der gleichen Untersuchung auch hervorgeht, dass Betriebsräte von Dezentralisierung dann eher positive Effekte erwarten, wenn sie selbst einem Betrieb ohne Tarifvertrag angehören.

Wie die hier präsentierten Schätzergebnisse belegen, ist es einer beachtlichen Zahl von Betriebsräten durchaus gelungen, zumindest die aus der „kontrollierten“ Dezentralisierung resultierenden Herausforderungen positiv zu bewältigen. Wie sich auch schon in den oben zitierten Einschätzungsfragen der Untersuchung andeutete, ist betriebliche Machtausübung kein Nullsummenspiel. Zwar erwarteten hier Betriebsräte, wie bereits ausgeführt, als Folge der Dezentralisierung einen Machtzuwachs des Arbeitgebers. Gleichzeitig aber hielten sich die Anteile der Betriebsräte, die einen Einflussverlust des Betriebsrates voraussahen, nahezu die Waage mit jenem Anteil, der vom genauen Gegenteil, nämlich der Einflusszunahme ausgeht (Bispinck 2005, S. 305). Die hier präsentierten Daten liefern keine Anzeichen dafür, dass sich kontrollierte Dezentralisierung als durchschlagkräftige Partizipationsbremse erweist. Dies kann zweierlei bedeuten: Nach einer ersten Interpretation vermögen Betriebsräte den von kontrollierter Dezentralisierung ausgehenden Druck halbwegs produktiv zu bewältigen und die ihnen aus der Anwendung von Öffnungsklauseln zuwachsenden Aufgaben immerhin so erfolg- 
reich zu übernehmen, dass die Effektivität der Betriebsratsarbeit nicht leidet. Eine zweite Interpretation dreht den Kausalzusammenhang um. Demnach lassen sich überhaupt nur starke und effektive Betriebsräte auf die Anwendung von Öffnungsklauseln ein. Trauen sich Betriebsräte die Bewältigung koordinierter Dezentralisierung nicht zu, vermögen sie sich als erfolgreicher Vetospieler in Szene zu setzen und widersetzen sich etwaigen Bestrebungen des Managements zur Anwendung von Öffnungsklauseln. Die Querschnittsanalyse vermag nicht zwischen Kausalrichtungen zu differenzieren, es bleibt aber dabei: Unabhängig davon, ob sich Betriebsräte nun als erfolgreiche Vetospieler in Szene setzen oder aber Anforderungen produktiv zu bewältigen vermögen, in der Mehrheit lässt sich (noch) kein lähmender Effekt der Öffnungsklauseln auf die Bewertung der
Betriebsratsarbeit nachweisen. Dies bedeutet allerdings nicht, dass alle von Öffnungsklauseln für das Tarifvertragssystem selbst ausgehenden Konsequenzen damit ad acta gelegt werden können. Nach wie vor gibt es gute Gründe anzunehmen, dass die den Wettbewerb regulierende Funktion des Tarifvertrages ebenso wie die Durchsetzungsstärke der Gewerkschaften von der Öffnung der Tarifverträge tangiert wird.

Die hier vorgestellte Analyse liefert Belege dafür, dass überall dort, wo Dezentralisierung „unkontrolliert“ bzw. „wild“ erfolgt, auch in Bezug auf die Betriebsratsarbeit die Dämme zu brechen drohen. Unkontrollierte Abweichungen vom Tarifvertrag sind recht deutlich mit verminderter (wahrgenommener) Effektivität der Betriebsratsarbeit assoziiert. Insofern verweisen die Befunde nachdrücklich auf die Notwendigkeit zur Differenzierung unter- schiedlicher Dezentralisierungsformen. Es darf die Prognose gewagt werden, dass nicht allein das Ausgangsniveau, von dem aus Dezentralisierung erfolgt, eine Rolle spielt, sondern ebenso die konkreten Formen und Ausprägungen der Dezentralisierungsprozesse selbst. Und das gilt vermutlich nicht allein in Deutschland. Insofern erscheint es - anders als zuletzt von Ilsøe et al. (2007) behauptet - fraglich, ob tatsächlich das deutsche duale System als solches schlecht vorbereitet ist, um mit den von Dezentralisierung ausgehenden Herausforderungen umzugehen. Die hier präsentierten Befunde deuten an, dass zumindest die Fähigkeit des Systems, auf Betriebsebene mit den genannten Herausforderungen umgehen zu können, bislang noch besser ist als angenommen.

\section{LITERATUR}

Artus, I. (2003): Die Kooperation zwischen Betriebsräten und Gewerkschaften als neuralgischer Punkt des Tarifsystems. Eine exemplarische Analyse am Beispiel Ostdeutschlands, in: Industrielle Beziehungen 2, S. $250-272$

Artus, I. (2004): Testfeld Ost? Betriebliche Interessenvertretung in Ostdeutschland - Konsequenzen für den Westen, in: WSI-Mitteilungen 5, S. 271-276

Behrens, M./Kädtler, J. (2008): Gesamtbetriebsräte: Neue Zuständigkeiten und die Folgen für die betrieblichen Arbeitsbeziehungen, in: WSI-Mitteilungen 6, S. 297-304

Bispinck, R. (2005): Betriebsräte, Arbeitsbedingungen und Tarifpolitik, in: WSI-Mitteilungen 6, S. 301-307

Bispinck, R. (2004): Kontrollierte Dezentralisierung der Tarifpolitik - Eine schwierige Balance, in: WSI-Miteilungen 5, S. 237-245

Bispinck, R./Schulten, T. (1999): Flächentarifvertrag und betriebliche Interessenvertretung, in: Müller-Jentsch, W. (Hrsg.): Konfliktpartnerschaft. Akteure und Institutionen der industriellen Beziehungen, München und Mering, S. 185-212

Bispinck, R./Schulten, T. (2003): Verbetrieblichung der Tarifpolitik? Aktuelle Tendenzen und Einschätzungen aus Sicht von Betriebs- und Personalräten, in: WSI-Mitteilungen 3, S. 157-166

Ferner, A./Hyman, R. (1992): Introduction, in: Ferner, A./Hyman, R. (Hrsg.): Industrial Relations in the New Europe, Oxford, S. XVI-XXXVIII Howell, C. (1999): Unforgiven. British Trade Unionism in Crisis, in: Martin, A./Ross, G. (Hrsg.): The Brave New World of European Labor, European Trade Unions at the Millenium, New York/Oxford, S. 26-74
Ilsøe, A:/Madsen, L. S./Due, J. (2007): Impacts of Decentralisation Erosion or Renewal? The Decisive Link between Workplace Representation and Company Size in German and Danish Industrial Relations, in: Industrielle Beziehungen 3, S. 201-222

Katz, H. (1993): The Decentralizatin of Collective Bargaining: A Literature Review and Comparative Analysis, in: Industrial and Labor Relations Review 1, S. 3-22

Kochan, T. A./Katz, H. C./McKersie, R. B. (1986): The Transformation of American Industrial Relations, New York

Massa-Wirth, H. (2007): Zugeständnisse für Arbeitsplätze? Konzessionäre Beschäftigungsvereinbarungen im Vergleich Deutschland - USA, Berlin Massa-Wirth, H./Seifert, H. (2004): Betriebliche Bündnisse für Arbeit nur mit begrenzter Reichweite?, in: WSI-Mitteilungen 5, S. 246-254 Nienhüser, W./Hoßfeld, H. (2007): Regulierungsform und Verbetrieblichungsneigung von Managern und Betriebsräten. Ergebnisse einer empirischen Erhebung, in: Industrielle Beziehungen 4, S. 289-315

Rehder, B. (2006): Legitimitätsdefizite des Co-Managements. Betriebliche Bündnisse für Arbeit als Konfliktfeld zwischen Arbeitnehmern und betrieblicher Interessenvertretung, in: Zeitschrift für Soziologie 3, S. 227242

Rehder, B. (2003): Betriebliche Bündnisse für Arbeit in Deutschland. Mitbestimmung und Flächentarif im Wandel, Frankfurt/New York Rosenbaum, W. (1982): Die Wirkungen des Arbeitsrechts auf die Beziehungen zwischen Arbeitnehmern und Arbeitgebern im Betrieb, in: Leviathan 3, S. 393-423 
Schmidt, R./Trinczek, R. (1986): Erfahrungen und Perspektiven gewerkschaftlicher Arbeitszeitpolitik, in: Prokla 64, S. 85-108

Schnabel, C. (2006): Verbetrieblichung der Lohnfindung und der Festlegung von Arbeitsbedingungen, Arbeitspapiere der Hans-Böckler-Stiftung 118

Schroeder, W./Weinert, R. (1999): Anmerkungen zum Wandel industrieller Beziehungen in Deutschland. Kontrollierte oder unkontrollierte Dezentralisierung, in: Zeitschrift für Politikwissenschaft 4, S. 1295-1317

Seifert, H./Massa-Wirth, H. (2005): Pacts for employment and competitiveness in Germany, in: Industrial Relations Journal 3, S. 217-240

Swenson, P. A. (2002): Capitalists against Markets. The Making of Labor Markets and Welfare States in the United States and Sweden, Oxford Swenson, P. A./Pontusson, J. (2000): The Swedish Employer Offensive against Centralized Wage Bargaining, in: Iversen, T./Pontusson, J./Soskice, D. (Hrsg.): Unions, Employers, and Central Banks, Macroeconomic Coordination and Institutional Change in Social Market Economies, Cambridge, S. 77-106
Thelen (1991): Union of Parts. Labor Politics in Postwar Germany, Ithaca/ NY

Traxler, F. (2003): Bargaining (De)centralization, Macroeconomic Performance and Control over the Employment Relationship, in: British Journal of Industrial Relations 1, S. 1-27

Traxler, F. (1995): Farewell to labour market associations? Organized versus disorganized decentralization as a map for industrial relations, in: Crouch, C./Traxler, F. (Hrsg.): Organized Industrial Relations in Europe: What Future?, Aldershot, S. 3-19

Weltz, F. (1977): Kooperative Konfliktverarbeitung, in: Gewerkschaftliche Monatshefte 28, S. 291-301

Wittemann, K. P. (1994): Ford-Aktion. Zum Verhältnis von Industriesoziologie und IG Metall in den sechziger Jahren, Marburg

Zachert, U. (1997): Modernisierung oder Liquidation der Tarifautonomie?, in: Kritische Justiz 4, S. 411-428 